\title{
Changes in tactile discrimination and in visual reaching after successive and simultaneous bilateral posterior parietal ablations in the monkey
}

\author{
G. ETTLINGER AND J. E. KALSBECK ${ }^{1}$ \\ From the Institute of Neurology, Queen Square, London
}

Recent quantitative studies of behaviour changes in the monkey following posterior parietal ablations have been reviewed by Bates and Ettlinger (1960). Various kinds of impairment (predominantly affecting tactile discrimination and the accuracy of reaching) have been recorded as a result of such bilaterally symmetrical ablations. Similar impairment has not been looked for in animals with unilateral posterior parietal ablations, except by Ruch and Fulton (1935) in one animal.

With a unilateral posterior parietal ablation a defect restricted to the contralateral limbs, a bilateral defect, or no defect at all are possible results. If subsequently the corresponding posterior parietal ablation is made from the other hemisphere we might expect any contralateral effect of the first operation to be re-duplicated in mirror fashion; or any bilateral defects to be accentuated; or even some new defect to become manifest where none had previously been observed.

To explore these possibilities we have studied the effect of successive unilateral posterior parietal removals in eight monkeys. Four of these received a right ablation followed by a second ablation on the left. Four other animals received a left followed by a right removal. All eight animals were studied pre-operatively, between their first and second operation, and also after the second operation by clinical and quantitative methods. All animals were given at least two systematic neurological examinations after each operation; their performance with one (the left) hand on a test of tactile shape discrimination in the dark was evaluated quantitatively; their ability to reach accurately with the left hand for small moving targets in the light was assessed. Three additional animals received bilaterally symmetrical posterior parietal ablations at a single

${ }^{1}$ Fellow of the National Institutes of Health, Bethesda, U.S.A. (under grant BT-480). operation and were studied in the same way before and after their only operation.

\section{METHODS \\ SUBJECTS}

Fourteen previously untrained rhesus monkeys (weight range 3.25 to $6.25 \mathrm{lb}$.) were used. Three animals (Nos. 20 i 9, and 13) had received dietary supplements of Tryptophand for a period of 30 days ending one week before training was started. The 14 animals were divided at random into two groups for investigation during two consecutive periods, owing to limitation of facilities. Animals Nos. 1 응 $3,5,6,7$, and 14 formed group I: Nos. 2, 4, 8, 9, 10 它 11,12 , and 13 formed group II. Four animals, Nos. 1 to 4 received right followed by left posterior parietal ablations $s \vec{\circ}$ Four animals, Nos. 5 to 8, received left followed by right ${ }^{+}$N posterior parietal ablations. Three animals, Nos. 9 to 11, sustained one-stage bilateral posterior parietal removals. Animals Nos. 12 and 13 were given respectively right and left anterior parietal ablations; animal No. 14 received a somewhat larger than intended right posterior parietal ablation.

\section{OPERATIONS}

Details of operative procedure and of the extent of the posterior parietal removals are to be found elsewhere (Bates and Ettlinger, 1960). In brief, the posterior parietal ablations extended anteriorly on the lateral surface from the lunate sulcus to an approximately dorso-ventral line bisecting the intraparietal sulcus. Small portions of superior temporal and of preoccipital cortex were removed. The ablations were carried medially down to the corpus callosum.

The anterior parietal removals extended posteriorly from the central sulcus to a dorso-ventral line passing through the junction of the posterior one-third and anterior two-thirds of the intraparietal sulcus. The inferior boundary of the removal extended from the foot of the central sulcus posteriorly to the Sylvian fissure, and then along the fissure. Medially, the ablations were carried 
down to the corpus callosum. These anterior removals were designed to be of the same size as the posterior parietal ablations. All left-sided removals were made slightly larger than the corresponding right-sided lesions. Care was taken to remove all grey matter from the depths of sulci situated in the area of the lesion.

\section{HISTOLOGICAL PROCEDURES AND FINDINGS}

When each animal had been completely studied it was anaesthetized and perfused with saline and formalin, and its brain was removed. All brains were photographed from six views (anterior, posterior, dorsal, ventral, left lateral, and right lateral). The hemispheres of six brains were separated by a cut in a mid-saggital plane and additional photographs were taken of the medial surfaces. Serial coronal sections of $25 \mathrm{~m} \mu$ thickness were cut from the other eight brains. Every twentieth section was stained with thionine. Diagrams of the extent of the lesions in all animals (except for No. 14) are presented in Fig. 1; and representative coronal sections through the
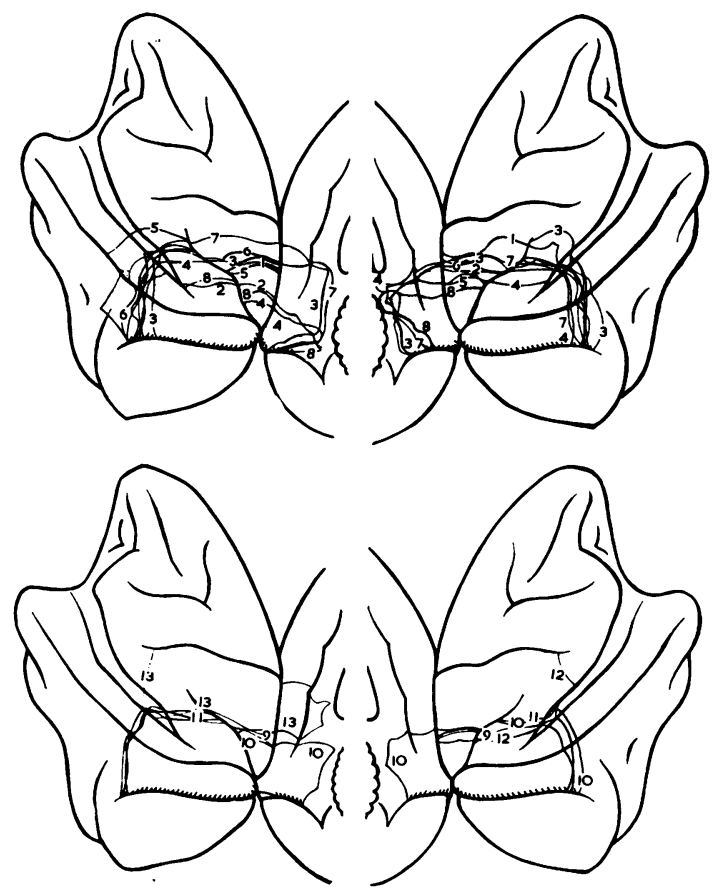

FIG. 1. Outline diagrams, based upon post-mortem inspection and photographic evidence, of the extent of the cortical removals in animals Nos. 1 to 13. All diagrams are charted on one 'standard' brain representation, showing left lateral, left medial, right medial, and right lateral surfaces. Diagrams for animals having successive posterior removals are shown in the upper part; for animals having one-stage bilateral posterior removals or unilateral anterior removals in the lower part. Removals from the medial surface are shown only for six animals (Nos. 3, 4, 7, 8, 10, and 13) in which the hemispheres were separated. area of the lesions for animals Nos. 1, 2, 5, 6, 9, 11, and 12 in Fig. 2.

Retrograde degeneration in the thalamus was studied in six animals (Nos. 1, 2, 5, 6, 9, and 11) with posterior parietal ablations. Changes were seen in the nucleus pulvinar lateralis, the nucleus lateralis posterior, and the nucleus geniculate lateralis as previously described by Bates and Ettlinger (1960). In addition degeneration was found in the nucleus pulvinar inferior on both sides in animals Nos. 1 and 6 and in the nucleus ventralis posterolateralis on the left side only in animals Nos. 5 and 6.

\section{NEUROLOGICAL EXAMINATION}

All animals were examined pre-operatively; after each operation every animal was examined on the sixth day and again on the tenth post-operative day. Animals were examined in a special chair with restraints only about the neck and abdomen. Neurological examinations of all animals were performed in a systematic manner, according to a predetermined scheme. To evaluate immediate post-operative effects the animals were observed in their cages.

Eye movements, head movements, visual fields, reaction to sounds, reaction to threat, tone, tactile and visual placing were examined as previously described by Bates and Ettlinger (1960). During examinations for visual placing the two half fields were not individually tested, i.e., edges of surfaces were present in both half fields. Placing reactions were considered to be reduced if repeated stimulation gave rise to only a delayed and feeble reaction.

Somatic sensation was tested by light touch with an artist's brush, a jet of air from a syringe, and by prick with a sharp needle; responses to simultaneous bilateral stimulation, visual and tactile, were observed. Localization without vision of a clip applied to the lateral aspect of each lower limb was tested with each hand separately.

Reactions to palmar touch were induced separately with a light stationary and a distally moving stimulus (the examiner's index finger). In animals of group I the predominant plantar response was recorded. In animals of group II, 10 consecutive plantar responses of each side were noted at each examination. The leg was held relaxed in approximately $90^{\circ}$ flexion at the hip, knee, and ankle joints and all movements initiated during light stimulation with a narrow blunt object along the lateral plantar surface and across medially towards the hallux were recorded.

To test the accuracy of reaching with vision unobstructed the examiner held a small food object consecutively in various sectors of the animal's visual field; again the food was placed in the animal's one hand which was held by the examiner in the different sectors of the field of vision; both left and right hands, and left and right half fields of vision were individually tested. Reaching without visual control was tested by placing the food object in one of the animal's hands and restraining this hand in a position either out of the field of vision (too far to the left or right) or obstructed from view by an opaque object. 

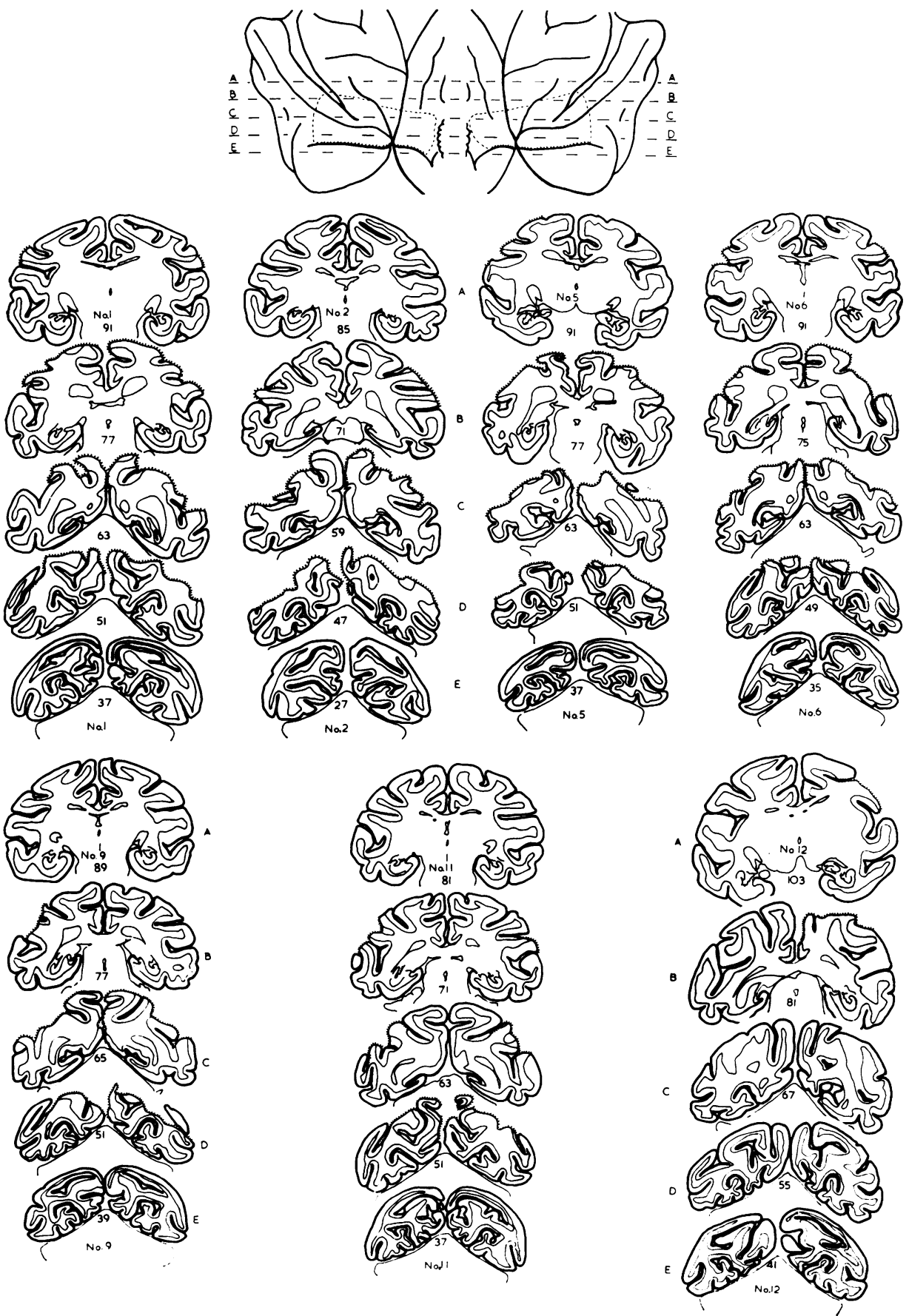

FIG. 2. Cross-sections, at about the levels $A$ to $E$ shown on the brain diagram, through the areas of removal for animals Nos. 1, 2, 5, 6, 9, 11, and 12. The left half of each cross-section refers to the left hemisphere. 


\section{QUANTITATIVE TEST PROCEDURES}

These are discussed under apparatus, test objects, and training.

Apparatus Animals were trained in standard apparatus which has been previously described (Bates and Ettlinger, 1960). With this apparatus the animal could make discriminations either by vision if the shelf on which the test objects were placed was illuminated, or by touch if the light was extinguished. Two infra-red sources illuminated the shelf from above, so that even in darkness it was possible to observe the animal's movements by means of an infra-red telescope.

Test objects For discrimination training, two black boxes (each measuring 4.5 by 2.5 by 2 in. high) were placed on the shelf, side by side, and 12 in. apart from centre to centre. The shapes were fastened to the lids of these boxes, being set slightly forward, i.e., towards the animal, from the centres of the lids, and the animals directly grasped them in pushing open the lids. In the tactile shape discrimination test the animals were required to discriminate between the solid figures $L$ ( 1.4 by 1.25 in. outer dimensions, 0.35 in. wide, and $0.5 \mathrm{in}$. deep), and $\mathrm{T}(1.8 \mathrm{in}$. across, $1.4 \mathrm{in}$. high, $0.35 \mathrm{in}$. wide, and $0.5 \mathrm{in}$. deep). Choice of the former was rewarded.

For the quantitative test of visual reaching a small white sugar pellet $(0 \cdot 1 \mathrm{~g}$. and $0 \cdot 2 \mathrm{in}$. diameter) was placed upon a dark grey moving belt. This belt was 17.5 in. long and $0.9 \mathrm{in}$. wide, and it moved at an average rate of $1.6 \mathrm{in}$. per second across the shelf to left or right, at a distance of 6 in. from the animal's cage (having horizontal bars). A constant-speed motor was used to drive the belt. However, the driving pulley was made slightly elliptical so that there were small systematic variations in the rate of movement.

Training procedures and sequence of training During discrimination training animals were given 40 trials a day. Training was always continued until the animal reached a standard level of proficiency. This consisted of making no more than 10 errors in 100 consecutive trials. The test score represents the number of trials, in excess of 100 , required to achieve the standard level of performance (10 or fewer errors in 100 trials). The position, to left or right, of the positive shape was balanced. Mistakes were not corrected. In the event of severe position habits the procedure described by Bates and Ettlinger (1960) was used. The reward for a correct response consisted of a sugar pellet $(0.5 \mathrm{~g}$.) for animals of group I, and of a peanut for those of group II.

During quantitative testing for accuracy of visual reaching the target was repeatedly presented until the animal had attempted to grasp it on 25 occasions, i.e., for 25 trials, on each of two days. If the animal did not attempt to reach during any of 25 presentations on each of five days (as happened pre-operatively with animals Nos. 8 and 10) testing was abandoned. The sugar pellet was placed at one end of the belt and the direction of its movement to the left or right was determined by a random procedure. The animal's head and eye movements were unrestricted. A trial consisted of an attempt made by the animal to grasp the sugar pellet. It was scored as a failure if the pellet was knocked off the belt, if the pellet was missed so badly that it moved across the shelf without being touched, or if the pellet was grasped but was subsequently allowed to fall from the hand before reaching the mouth. Other trials were scored as accurate. However, post-operatively for animals of group I and at all stages for animals of group II a further distinction was made between, on the one hand, those trials scored as accurate in which the pellet was grasped accurately at the first attempt, and, on the other hand, trials (also scored as accurate) in which it was grasped accurately only at the second or subsequent attempts.

All animals were first trained on simple adaptation tests of texture discrimination in the light and then in the dark. They were allocated to their respective operative groups (right-left, left-right, bilateral, posterior parietal, or anterior parietal) on the basis of their scores on these preliminary tests. The summed scores for the individual animals are:

Nos. $1-190 ; 2-230 ; 3-290 ; 4-480$ trials

Nos. 5-200; 6-260; 7-430;8-490 trials

Nos. $9-230 ; 10-280 ; 11-350$ trials

Nos. $12-330 ; 13$-not tested; $14-250$ trials

During this preliminary training the animals were accustomed to having a rubber ball filled with lead (weight of $1.25 \mathrm{lb}$.) attached by a bracelet to the right wrist. This served to restrict the use of the right hand to supporting the animal. The ball and bracelet were removed in between test sessions.

Animals were next taught (pre-operative learning) to discriminate in the dark with their left hand between the shapes $L$ and $T$. As each animal achieved the standard level of performance on this test it was allowed 14 days' rest, except that 50 trials (pre-operative testing) were given on the quantitative test of visual reaching during this rest period. After the 14 days' rest period the animal was retrained on the tactile shape discrimination test (pre-operative relearning) and then immediately received its first operation. A further period of 14 days' rest was allowed after surgery, except that 50 further trials (interor post-operative testing) were given on the quantitative test of visual reaching, starting on the ninth post-operative day. After the initial post-operative rest period the animal was retrained on the tactile shape discrimination test (inter- or post-operative relearning). If it relearnt this test without impairment (that is, did not require more than 10 trials, the maximum needed during pre-operative relearning) the animal immediately received its second operation (if a second operation was to be performed). If, however, its performance was impaired after the first operation a second inter-operative rest period of 14 days was allowed (with 50 further trials at visual reaching) and a second period of inter-operative retraining was given before the second operation. Another period of 14 days' rest was allowed after the second operation, except that again 50 trials were given on the test of visual reaching during this period. After the 14 days' rest period subsequent to the second operation the animal was once more retrained on the tactile shape discrimination. The training histories of all 14 animals are shown in detail in Table I. 
TABLE I

SEQUENCE OF TRAINING AND OF OPERATIONS FOR ALL ANIMALS

\begin{tabular}{|c|c|c|c|c|c|c|c|c|c|c|c|c|c|c|}
\hline Animal & 1 & 2 & 3 & 4 & 5 & 6 & 7 & 8 & 9 & 10 & 11 & 12 & 13 & 14 \\
\hline Learning ( $L$ vs $T$ ) & 300 & 200 & 400 & 520 & 260 & 540 & 330 & 590 & 390 & 450 & 360 & 280 & - & 240 \\
\hline $\begin{array}{l}\text { (Reaching) } \\
\text { Relearning (L vs T) } \\
\text { First operation }\end{array}$ & $\begin{array}{c}\text { (98) } \\
0 \\
\text { R. U }\end{array}$ & $\begin{array}{c}(100) \\
10 \\
\text { ilateral }\end{array}$ & $\begin{array}{c}\text { (96) } \\
0 \\
\text { ostr. }\end{array}$ & $\begin{array}{c}(98) \\
0\end{array}$ & $\begin{array}{c}(100) \\
0 \\
\text { L. U }\end{array}$ & $\begin{array}{c}(96) \\
0 \\
\text { ilateral }\end{array}$ & $\begin{array}{c}\text { (92) } \\
0 \\
\text { Postr. }\end{array}$ & - & $\begin{array}{c}\text { (96) } \\
0 \\
\text { Bilat }\end{array}$ & $\begin{array}{r}- \\
0 \\
\text { al Pos }\end{array}$ & $\begin{array}{c}(98) \\
0\end{array}$ & $\begin{array}{c}\text { (96) } \\
0 \\
\text { R. An }\end{array}$ & $\frac{(98)}{\mathrm{L} .}$ & $\begin{array}{c}(88) \\
10 \\
\text { Right }\end{array}$ \\
\hline $\begin{array}{l}\text { (Reaching) } \\
\text { Relearning (L vs } T \text { ) }\end{array}$ & $\begin{array}{l}(84) \\
210\end{array}$ & $\begin{array}{c}(86) \\
0\end{array}$ & $\begin{array}{l}(40) \\
110\end{array}$ & $\begin{array}{c}(98) \\
60\end{array}$ & $\begin{array}{c}(96) \\
10\end{array}$ & $\begin{array}{c}(100) \\
0\end{array}$ & $\begin{array}{c}(94) \\
10\end{array}$ & $\overline{0}$ & $\begin{array}{l}(62) \\
160\end{array}$ & $\overline{0}$ & $\begin{array}{c}(98) \\
40\end{array}$ & $\begin{array}{l}(0) \\
650+\end{array}$ & $\stackrel{(98)}{-}$ & $\begin{array}{c}(0) \\
650+\end{array}$ \\
\hline $\begin{array}{l}\text { (Reaching) } \\
\text { Relearning (L vs T) } \\
\text { Second operation }\end{array}$ & $\begin{array}{c}(96) \\
0 \\
\text { L. } \mathrm{Ur}\end{array}$ & - & $\begin{array}{c}(90) \\
0 \\
\text { ostr. }\end{array}$ & $\begin{array}{c}(96) \\
0\end{array}$ & $\overline{\text { R. U }}$ & - & - & - & $\begin{array}{c}(96) \\
0\end{array}$ & - & $\begin{array}{c}(100) \\
0\end{array}$ & $\underline{(0)}$ & - & $\underline{(0)}$ \\
\hline $\begin{array}{l}\text { (Reaching) } \\
\text { Relearning (L vs T) }\end{array}$ & $\begin{array}{c}(96) \\
10\end{array}$ & $\begin{array}{c}\text { (96) } \\
0\end{array}$ & $\begin{array}{c}(92) \\
10\end{array}$ & $\begin{array}{c}(96) \\
0\end{array}$ & $\begin{array}{c}(88) \\
0\end{array}$ & $\begin{array}{l}(70) \\
280\end{array}$ & $\begin{array}{l}(96) \\
310\end{array}$ & $\overline{50}$ & & & & & & \\
\hline $\begin{array}{l}\text { (Reaching) } \\
\text { Relearning (L vs } \mathrm{T} \text { ) }\end{array}$ & - & - & - & - & - & $\begin{array}{c}(94) \\
0\end{array}$ & $\begin{array}{c}(100) \\
0\end{array}$ & $\overline{0}$ & & & & & & \\
\hline
\end{tabular}

The figures not in brackets refer to the number of trials in excess of 100 required to reach the standard level of performance (10 or fewer errors in $\mathbf{1 0 0}$ trials) with the left hand on the tactile shape discrimination test ( $\mathrm{L}$ vs $\mathrm{T}$ ). The figures in brackets refer to the percentage accurate responses with the left hand in $\mathbf{5 0}$ trials on the quantitative test of visual reaching. The symbol + indicates that the animal failed to reach the standard level of performance within the stated number of trials; -indicates that relearning was not undertaken at this stage. Horizontal lines indicate periods of 14 days' rest. L signifies left-sided; R, right-sided; Postr, posterior parietal ablations; Antr, anterior parietal control ablations.

\section{RESULTS}

\section{PRE-OPERATIVE TESTING}

NEUROLOGICAL EXAMINATION All animals were examined before surgery to establish a standard of comparison for the post-operative examinations. Variability (both in the same animal and between animals) was noted in reactions to touch, to a puff of air, and to pin-prick. The plantar responses were not consistent (see Table II). The reaction to palmar touch was grasping, no reaction, or avoiding. The visual and tactile placing reactions were always present pre-operatively although somewhat delayed in one animal. Reaching for objects which could be seen was accurate; two animals were slightly inaccurate at reaching for food placed on one hand and held out of sight. All animals rapidly localized with either hand a clip applied to the skin of either leg.

QUANTITATIVE TESTS Reference is made to the preoperative test results when considering the effects of various operations.

\section{EFFECT OF UNILATERAL POSTERIOR PARIETAL ABLATIONS}

IMMEDIATE CHANGES Animals which received unilateral posterior parietal ablations (Nos. 1 to 8) were climbing about the cage and eating two hours after first sitting up. None of these eight animals showed a visual deficit. For the first few days the animals often sat facing away from the cage door or clinging to the back of the cage. They showed no preference for either side of the cage regardless of the laterality of the lesion. Climbing and jumping were only slightly clumsy.

NEUROLOGICAL EXAMINATION When examined after surgery no animal showed defects on testing the visual fields, eye or head movement, or the reaction to sounds.

Muscle tone was transiently decreased in the contralateral limbs of four animals (Nos. 1, 2, 3, and 5) on only the first examination. Two animals (Nos. 3 and 7) held the contralateral upper limb in a position of marked flexion at the elbow and wrist with extension of the fingers. There was also a reduction of spontaneous movement in the limb. Knee and ankle reflexes were equal on the two sides. Plantar reactions (Table II) were variable as were the reactions to palmar touch.

No animal showed a decreased reaction to touch, a puff of air, or to pin-prick on the side contralateral to the ablation. However, two animals were more reactive to sensory stimulation on the side opposite the lesion.

Three animals (Nos. 1, 3, and 7) showed varying degrees of inaccuracy in reaching with the contralateral hand (Table III) for objects that could be seen. Their errors were mostly medial to or beyond the object. Repeated attempts to grasp the object became progressively more inaccurate until finally the animal would no longer reach. On the second examination six out of the eight animals (Nos. 1, 2, 
TABLE II

PLANTAR RESPONSE IN EIGHT ANIMALS (GROUP II) BEFORE AND AFTER SURGERY

\begin{tabular}{ccccccc}
$\begin{array}{l}\text { No } \\
\text { Reaction }\end{array}$ & $\begin{array}{l}\text { Flexion } \\
\text { Alone }\end{array}$ & $\begin{array}{l}\text { Flexion } \\
\text { Followed by } \\
\text { Extension }\end{array}$ & $\begin{array}{l}\text { Extension } \\
\text { Alone }\end{array}$ & $\begin{array}{l}\text { Extension } \\
\text { (digits 2-5 } \\
\text { only) }\end{array}$ & $\begin{array}{l}\text { Extension } \\
\text { (digits 2-5 } \\
\text { and adduction } \\
\text { of hallux) }\end{array}$ & $\begin{array}{l}\text { Total No. of } \\
\text { Responses } \\
\text { Observed }\end{array}$ \\
\hline$\%$ & $\%$ & $\%$ & $\%$ & $\%$ & $\%$ & \\
26 & 12 & 3 & 39 & 11 & 9 & 160
\end{tabular}

\begin{tabular}{|c|c|c|c|c|c|c|c|}
\hline Pre-operative & $\begin{array}{l}\% \\
26\end{array}$ & $\begin{array}{l}\% \\
12\end{array}$ & $\begin{array}{r}\% \\
3\end{array}$ & $\begin{array}{l}\% \\
39\end{array}$ & $\%$ & $\begin{array}{c}\% \\
9\end{array}$ & 160 \\
\hline \multicolumn{8}{|c|}{ Successive Unilateral Posterior Parietal Ablations } \\
\hline $\begin{array}{l}\text { Ipsilateral } \\
\text { Operation I + } 6 \text { days }\end{array}$ & 17 & 30 & & 33 & & 20 & 30 \\
\hline Contralateral & 53 & 10 & & 30 & 3 & 3 & 30 \\
\hline $\begin{array}{l}\text { Ipsilateral } \\
\text { Operation } I+10 \text { days }\end{array}$ & & & & 100 & & & 30 \\
\hline Contralateral & 30 & 3 & & 57 & 7 & 3 & 30 \\
\hline $\begin{array}{l}\text { Ipsilateral } \\
\text { Operation II + } 6 \text { days }\end{array}$ & 7 & 10 & 13 & 70 & & & 30 \\
\hline Contralateral & 7 & 10 & & 60 & & 23 & 30 \\
\hline $\begin{array}{l}\text { Ipsilateral } \\
\text { Operation II }+10 \text { days }\end{array}$ & 20 & & 10 & 57 & & 13 & 30 \\
\hline Contralateral & 7 & 7 & & 86 & & & 30 \\
\hline \multicolumn{8}{|c|}{ One-stage Bilateral Posterior Parietal Ablations } \\
\hline $\begin{array}{l}\text { Operation }+6 \text { days } \\
\text { Operation }+10 \text { days }\end{array}$ & $\begin{array}{l}45 \\
28\end{array}$ & $\begin{array}{l}3 \\
2\end{array}$ & & $\begin{array}{l}27 \\
70\end{array}$ & 25 & & $\begin{array}{l}60 \\
60\end{array}$ \\
\hline \multicolumn{8}{|c|}{ Unilateral Anterior Parietal Ablations } \\
\hline $\begin{array}{l}\text { Ipsilateral } \\
\text { Operation }+6 \text { days } \\
\text { Contralateral }\end{array}$ & $\begin{array}{r}5 \\
100\end{array}$ & & 5 & 60 & 25 & 5 & $\begin{array}{l}20 \\
20\end{array}$ \\
\hline $\begin{array}{l}\text { Ipsilateral } \\
\text { Operation }+10 \text { days } \\
\text { Contralateral }\end{array}$ & 100 & & 5 & 95 & & & 20 \\
\hline
\end{tabular}

The figures in the table are per cent responses conforming to one of the six possible types.

$3,5,6$, and 7) were inaccurate in reaching for food held out of the animal's sight in the ipsilateral hand. Five also showed a defect in reaching for food similarly held in the contralateral hand.

Placing reactions were reduced or absent only on the side opposite the lesion. Either the upper or the lower or both limbs were affected in seven of the eight animals (not in No. 7). Tactile placing was more severely and more permanently affected than was visual placing.

In summary, only contralateral changes, affecting predominantly the accuracy of reaching and the placing reactions, were observed.

QUANTITATIVE TESTS These were done to test changes when the first operation was contralateral to the hand tested and when it was ipsilateral.

Contralateral to the hand tested Before surgery no animal required more than 10 trials to relearn with the left hand the tactile shape discrimination test to the standard level of performance after an interval of 14 days' rest. The range of the number of trials required to relearn the discrimination following ablation of only the right (contralateral) posterior parietal region (animals Nos. 1 to 4, Table IV) is
0 to 210 trials. Therefore a first contralateral posterior parietal ablation gave rise to moderate impairment of tactile shape discrimination in three out of four animals which exceeded the pre-operative maximum.

Before surgery the performance scores of all animals ranged between 88 and $100 \%$ accurate responses with the left hand on the quantitative test of visual reaching. The comparable range after ablation of only the right (contralateral) posterior parietal region (animals Nos. 1 to 4 , Table IV) is 40 to $98 \%$ accurate responses. Therefore a first contralateral posterior parietal ablation also caused impaired accuracy of visual reaching in three out of four animals which gave scores below the preoperative minimum.

Ipsilateral to the hand tested The range of the number of trials required to relearn with the left hand the tactile shape discrimination after ablation of only the left (ipsilateral) posterior parietal region (animals Nos. 5 to 8 , Table $\mathrm{V}$ ) is 0 to 10 trials. Therefore a first ipsilateral posterior parietal ablation gave rise to no clear impairment of tactile shape discrimination since no animal exceeded the pre-operative maximum. The difference between the 
TABLE III

DEFECTS OF REACHING IN ANIMALS 1 TO 13 BEFORE AND AFTER SURGERY

\begin{tabular}{|c|c|c|c|c|}
\hline & With Vision & $\begin{array}{l}\text { With Vision (Object } \\
\text { in Restrained Hand) }\end{array}$ & $\begin{array}{l}\text { Without Vision (Object } \\
\text { in Restrained Hand) }\end{array}$ & $\begin{array}{l}\text { Total Hands } \\
\text { Tested }\end{array}$ \\
\hline Pre-operative & & $1 /$ & $\mathbf{X X}$ & 26 \\
\hline
\end{tabular}

Successive Unilateral Posterior Parietal Ablations

Ipsilateral
Operation $I+6$ days

Contralateral

II $/ 1$

$1 /$

Ipsilateral

II $\quad \mathbf{x \times x} \quad$ /III

IIII $\mathbf{x}$

IIII

Operation $I+10$ days

Contralateral

Ipsilateral

Operation II +6 days

Contralateral

$\mathrm{XXX}$

$\mathrm{XXX} \quad \mathrm{XXX}$

Ipsilateral
Operation II +10 days
Contralateral

$\mathbf{X}$

One-stage Bilateral Posterior Parietal Ablations

Operation +6 days

Operation +10 days

$\begin{array}{ll}1 & \mathbf{x} \\ 1 & \mathbf{x}\end{array}$

Unilateral Anterior Parietal Ablations

Ipsilateral

Operation +6 days

Contralateral

Ipsilateral

Operation +10 days

Contralateral

$x$

XXX

$\mathbf{X}$

$\mathbf{X X X} \quad$ /

$\mathbf{X}$

$\mathbf{X}$

$\mathbf{X X}$

8

Ipsilateral

Operation +21 days

Contralateral

$\mathrm{X}$

$x$

1

$\mathbf{x}$

XXXX

$\begin{array}{ll}\mathrm{XXX} & 8 \\ \mathrm{XXXX} & 8\end{array}$

$\begin{array}{ll}\mathbf{x} & 1 / \\ & 1 \\ \mathbf{x} & 1 \\ & \end{array}$

X

Each animal was tested, separately with each hand, under three conditions shown as column headings. Accurate reaching responses are not tabulated. Each symbol in the Table refers to the response of one hand of one animal. Thus the symbol/indicates that one animal did not attempt to reach with one hand. The symbol $X$ indicates that one animal reached inaccurately with one hand.

\section{TABLE IV}

EFFECT OF RIGHT (CONTRALATERAL) POSTERIOR PARIETAL ABLATION ON RELEARNING OF TACTILE SHAPE DISCRIMINATION TEST AND ON VISUAL REACHING

\begin{tabular}{|c|c|c|c|c|c|c|c|c|}
\hline \multirow[b]{2}{*}{ Animal } & \multicolumn{4}{|c|}{ After First Operation } & \multicolumn{4}{|c|}{ After Second Operation } \\
\hline & 1 & 2 & 3 & 4 & 5 & 6 & 7 & 8 \\
\hline $\begin{array}{l}\text { No. of trials to standard level of performance ( } \mathrm{L} \text { vs } \mathrm{T} \text {, tactile shapes) } \\
\text { Percentage accurate responses in } 50 \text { trials (visual reaching) }\end{array}$ & $\begin{array}{r}210 \\
84\end{array}$ & $\begin{array}{r}0 \\
86\end{array}$ & $\begin{array}{r}110 \\
40\end{array}$ & $\begin{array}{l}60 \\
98\end{array}$ & $\begin{array}{r}0 \\
88\end{array}$ & $\begin{array}{r}280 \\
70\end{array}$ & $\begin{array}{r}310 \\
96\end{array}$ & 50 \\
\hline
\end{tabular}

TABLE V

EFFECT OF LEFT (IPSILATERAL) POSTERIOR PARIETAL ABLATION ON RELEARNING OF TACTILE SHAPE DISCRIMINATION TEST AND ON VISUAL REACHING

\begin{tabular}{|c|c|c|c|c|c|c|c|c|}
\hline \multirow[b]{2}{*}{ Animal } & \multicolumn{4}{|c|}{ After Second Operation } & \multicolumn{4}{|c|}{ After First Operation } \\
\hline & 1 & 2 & 3 & 4 & 5 & 6 & 7 & 8 \\
\hline $\begin{array}{l}\text { No. of trials to standard level of performance ( } L \text { vs } T \text {, tactile shapes) } \\
\text { Percentage accurate responses in } 50 \text { trials (visual reaching) }\end{array}$ & $\begin{array}{l}10 \\
96\end{array}$ & $\begin{array}{r}0 \\
96\end{array}$ & $\begin{array}{l}10 \\
92\end{array}$ & $\begin{array}{r}0 \\
96\end{array}$ & $\begin{array}{l}10 \\
96\end{array}$ & $\begin{array}{r}0 \\
100\end{array}$ & $\begin{array}{l}10 \\
94\end{array}$ & 은 \\
\hline
\end{tabular}

scores after a first ipsilateral (animals Nos. 5 to 8) and a first contralateral (animals Nos. 1 to 4 ) posterior removal just fails to reach statistical significance ( $P=0.057)$. (The Mann-Whitney test is used in all statistical comparisons; ties between animals with identical learning scores are resolved by reference to the error scores.)
The range of the performance scores with the left hand on the quantitative test of visual reaching following ablation of only the left (ipsilateral) posterior parietal region (animals Nos. 5 to 7 , Table V) is 94 to $100 \%$ accurate responses. Therefore a first ipsilateral posterior parietal ablation gave rise to no impairment in the accuracy of visual 
reaching, since no animal obtained a score below the pre-operative minimum. Again the difference between the scores after a first ipsilateral (animals Nos. 5 to 7) and a first contralateral (animals Nos. 1 to 4) posterior removal fails to attain statistical significance $(P=0 \cdot 11)$.

In summary, contralateral but not ipsilateral removals caused impairment of tactile discrimination and of visual reaching.

\section{EFFECT OF TWO-STAGE BILATERAL POSTERIOR PARIETAL ABLATIONS}

IMMEDIATE CHANGES AND NEUROLOGICAL EXAMINATION The second (two-stage bilateral) posterior parietal ablations caused no change in behaviour during the immediate post-operative period and no neurological defect that had not been observed with the first (unilateral) ablations. There was no exacerbation of any deficits that followed the first ablation.

QUANTITATIVE TESTS These were repeated as after the first operations.

Contralateral to the hand tested The range of the number of trials required to relearn with the left hand the tactile shape discrimination when the right (contralateral) posterior parietal region was ablated after a left posterior parietal removal (animals Nos. 5 to 8, Table IV) is 0 to 310 trials. These scores again reflect variable impairment. Statistical comparison between the effects of a first contralateral ablation and of a comparable ablation subsequent to an ipsilateral removal indicates that the difference is not significant $(P=0.44)$.

The range of the performance scores with the left hand on the quantitative test of visual reaching when the right (contralateral) posterior parietal region was ablated subsequent to a left posterior parietal removal (Nos. 5 to 7, Table IV) is 70 to $96 \%$ accurate responses. These scores likewise reflect variable impairment. Statistical comparison between the effects of a first contralateral ablation and of a comparable ablation subsequent to an ipsilateral removal indicates again that the difference is not significant $(P=0.43)$.

The results relating to contralateral posterior ablations, whether as first or second operations, are shown for both tests in Table IV.

Ipsilateral to the hand tested The range of the number of trials required to relearn with the left hand the tactile shape discrimination when the left (ipsilateral) posterior parietal region was ablated subsequent to a right posterior parietal removal (Nos. 1 to 4 , Table V) is 0 to 10 trials. Statistical comparison between the effects of a first ipsilateral ablation and of a comparable ablation subsequent to a contralateral removal indicates that the difference is not significant $(P=0.37)$.

The range of the performance scores with the left hand on the quantitative test of visual reaching when the left (ipsilateral) posterior parietal region was ablated subsequent to a right posterior parietal removal (Nos. 1 to 4 , Table $V$ ) is 92 to $96 \%$ accurate responses. Statistical comparison between the effects of a first ipsilateral ablation and of a comparable ablation subsequent to a contralateral removal indicates again that the difference is not significant $(P=0 \cdot 37)$.

The results relating to ipsilateral posterior parietal ablations, whether as first or second operations, are shown for both tests in Table V.

In summary, second contralateral and ipsilateral operations gave rise to no more severe effects than the comparable first operations.

Difference between contralateral and ipsilateral operations In this comparison the significance of the difference between performance after contralateral and ipsilateral ablations was calculated for all eight animals (seven for reaching) disregarding the order of the operations (Wilcoxon's test). All animals were given one score reflecting the effect of a contralateral removal and another for the effect of an ipsilateral removal. The contralateral ablations are found to give rise to significantly more impairment than the ipsilateral ablations on the tactile shape discrimination test $(P=0.02$, one-tailed) and on the test for accuracy of visual reaching ( $P=$ $<0.05$, one-tailed).

A summary of the findings on the test of tactile shape discrimination has already been given elsewhere (Ettlinger and Kalsbeck, 1961).

\section{EFFECT OF ONE-STAGE BILATERAL POSTERIOR PARIETAL ABLATIONS}

IMMEDIATE CHANGES One of the three animals (Nos. 9 to 11) which received one-stage bilateral removals of the posterior parietal region showed a transient visual disturbance lasting several hours. Another animal on the day of the operation would bring his mouth to food instead of reaching for it but would place his hand in the drinking water and then lick it. On the day after surgery all animals were mildly lethargic and slightly clumsy. However, they were eating and moving about the cage.

NEUROLOGICAL EXAMINATION There were no differences between these three animals and those with similar removals made at two operations.

QUANTITATIVE TESTS The range of the number of trials required to relearn with the left hand the tactile 
shape discrimination following ablation of both left and right posterior parietal regions at one operation (Nos. 9 to 11 , Table VI) is 0 to 160 trials. These scores reflect variable impairment, since two out of three animals exceeded the pre-operative maximum. If now the scores of the three animals (Nos. 9 to 11) with one-stage bilateral removals are compared with those of four animals (Nos. 1 to 4 ) having first and contralateral removals and with those of four other animals (Nos. 5 to 8 ) having second and contralateral removals, the differences fail to reach significance ( $P=0.37$ and 0.31 respectively).

Only two animals (Nos. 9 and 11) could be tested on the quantitative test of visual reaching after onestage bilateral operations since animal No. 10 failed to respond even pre-operatively. These two animals gave scores of 62 and $98 \%$ accurate responses, the former falling below the pre-operative minimum. Inspection of the scores suggests that one-stage bilateral posterior parietal ablations have the same effect on visual reaching as either first and contralateral or second and contralateral posterior parietal removals.

\section{EFFECT OF OTHER OPERATIONS}

NEUROLOGICAL EXAMINATION Two further animals (Nos. 12 and 13) received ablations only of the anterior parietal area on one side so that their defect of reaching could be compared with that shown by animals having posterior parietal removals. A summary of the neurological results is given in Table VII, and only selected findings are further reported.

TABLE VI

EFFECT OF ONE-STAGE BILATERAL POSTERIOR PARIETAL ABLATION (ONLY OPERATION) ON RELEARNING OF TACTILE SHAPE DISCRIMINATION TEST AND ON VISUAL REACHING

Animal Percentage accurate responses in 50 trials (visual reaching)

$\begin{array}{rll}9 & 10 & 11 \\ 160 & 0 & 40 \\ 62 & - & 98\end{array}$

TABLE VII

RESULtS OF NEUROLOGICAL EXAMINATION IN TWO ANIMALS (NOS. 12 AND 13) BEFORE AND AFTER ANTERIOR PARIETAL ABLATIONS

\begin{tabular}{|c|c|c|c|c|c|c|c|}
\hline $\begin{array}{l}\text { Light } \\
\text { Touch }\end{array}$ & $\begin{array}{l}\text { Puff of } \\
\text { Air }\end{array}$ & Pin-prick & $\begin{array}{l}\text { Simultaneous } \\
\text { Stimuli }\end{array}$ & $\begin{array}{l}\text { Palmar } \\
\text { Touch } \\
\text { (stationary) }\end{array}$ & $\begin{array}{l}\text { Palmar } \\
\text { Touch } \\
\text { (moving) }\end{array}$ & $\begin{array}{l}\text { Visual } \\
\text { Placing }\end{array}$ & $\begin{array}{l}\text { Tactile } \\
\text { Placing }\end{array}$ \\
\hline$i$ & $i$ & $c$ & $i$ & $i$ & $i$ & $i$ & $i$ \\
\hline
\end{tabular}

Pre-operative

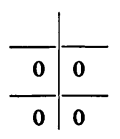

\begin{tabular}{l|l}
\hline 0 & 0 \\
\hline 0 & 0
\end{tabular}

\begin{tabular}{l|l}
\hline 0 & 0 \\
\hline 0 & 0
\end{tabular}

$=$

Operation +6 days

$$
\begin{array}{c|c} 
& \downarrow \downarrow \\
\hline 0 & 00 \\
\hline 0 & 00
\end{array}
$$$$
\begin{array}{c|c} 
& \downarrow \downarrow \\
\hline 0 & 00 \\
\hline 0 & 00
\end{array}
$$
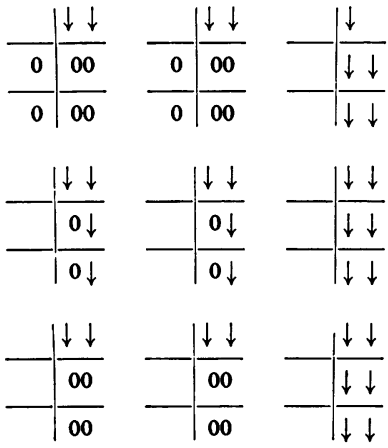

Normal responses are not indicated

$i$ Ipsilateral

0 No response

- Unable to test

G Grasping or flexion

= Equal response on the two sides c Contralateral

$\downarrow$ Decreased response

A Avoiding or extension

$>$ Greater than

$\begin{array}{llll}\mathbf{A} & \mathbf{A} & \mathbf{A} & \mathbf{A} \\ \mathbf{A} & \mathbf{0} & \mathbf{A} & \mathbf{0}\end{array}$
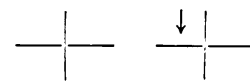

G 0

G 0

G $\mathbf{0}$

G $\mathbf{0}$

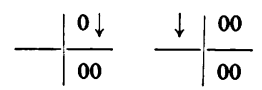

A 0

A 0

A $\mathbf{0}$

A $\mathbf{0}$

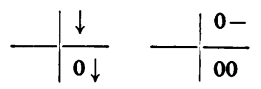

$\begin{array}{llll}\mathbf{A} & \mathbf{0} & \mathbf{A} & \mathbf{0} \\ \mathbf{A} & \mathbf{0} & \mathbf{A} & \mathbf{0}\end{array}$

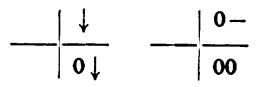


Reaching with the affected hand was severely impaired in both animals. They would reach medial to and beyond the object. Grasping and manipulation were very clumsy. Objects were usually held in the palm of the hand instead of in the normal way with the fingers. When bringing food to the mouth the animal would often also put a finger or part of the hand into the mouth.

Visual and tactile placing reactions (including crossed placing with the ipsilateral limb) were absent initially on testing the contralateral limbs. In both animals visual placing improved while tactile placing remained absent.

QUANTITATIVE TeSTS Animals Nos. 12 and 13 received anterior parietal ablations on the right side (contralateral to the hand tested) and left (ipsilateral) side respectively. The former animal was severely impaired on the test of tactile shape discrimination with the left hand. Initially it refused to respond in the dark. However, it learnt within 20 trials to discriminate without error when light was allowed. Twenty-six days after post-operative training began, that is, six weeks after surgery, it began to respond in the dark but failed to relearn the discrimination in 650 trials. Its performance on the test of visual reaching was likewise severely and persistently impaired (initial and terminal scores of $0 \%$ accurate). Animal No. 13 was tested only for the accuracy of visual reaching with the left hand, and was not impaired following an ipsilateral anterior removal (score of $98 \%$ accurate). Animal No. 14 with a larger than intended right (contralateral) posterior removal (extending into the postcentral gyrus) failed to relearn the tactile shape discrimination in 650 trials. It obtained scores of $0 \%$ accurate on the test of visual reaching both before and after its postoperative training on the tactile shape discrimination test.

\section{OTHER QUANTITATIVE RESULTS}

The performance scores of animals on the quantitative test of visual reaching were analysed in a second way (see section on Methods). For this further analysis a trial was scored as accurate only if a sugar pellet was grasped accurately at the first attempt. The results of this further analysis in relation to various posterior parietal removals are as follows:-

1 First and contralateral, mean $=59 \%$, vs second and contralateral operation, mean $=67 \%$ (not significant)

2 First and contralateral, mean $=59 \%$, vs first and ipsilateral operation, mean $=97 \%$ significant $(P=0.03)$.
3 First and ipsilateral, mean $=97 \%$, vs second and ipsilateral operation, mean $=87 \%$ significant $(P=0.03)$.

4 Second and contralateral, mean $=67 \%$, vs second and ipsilateral operation, mean $=87 \%$ significant $(\mathrm{P}=0.04)$.

The performance scores on the tactile shape discrimination test were also correlated with the accuracy of visual reaching for animals having posterior parietal ablations (Nos. 1 to 11). Animals Nos. 8 and 10 refused to reach for moving targets and so were excluded. Scores obtained after the contralateral operation were used for animals Nos. 1 to 7, after the bilateral removal for animals Nos. 9 and 11. The rank correlation between shape discrimination and reaching is found to be -0.27 , irrespective of the way in which performance at visual reaching was analysed (see above). This value is not significant for nine animals.

In addition, rank correlations were calculated between a measure of the anterior extension of the right-sided posterior parietal removals and performance respectively at tactile shape discrimination and at visual reaching. The right hemisphere lesions in animals Nos. 1 to 11 were first charted on standard brain diagrams. The greatest distance on the lateral surface between the lunate sulcus and the anterior margin of the removal was measured in a direction perpendicular to the lunate sulcus. With this measure the rank order (from largest to smallest extension) is as follows:-Animals Nos. 1, 3, 7, 6, 5, 2, 8, 4, 11, 10 , and 9. For the test of visual reaching scores representing percentage accurate responses irrespective of the number of attempts (see above) were used. The rank correlation between the anterior extent of the right-sided lesions and impairment at tactile shape discrimination is found to be 0.44 (not significant, $\mathrm{n}=11$ ); between the anterior extent of the right-sided lesions and the accuracy of visual reaching $-0 \cdot 32$ (not significant, $\mathrm{n}=9$ ).

Finally, an analysis was made of the laterality of the response errors of animals Nos. 1 to 8 on the tactile shape discrimination test. The numbers of left-sided and right-sided response errors, i.e., when the correct response was to the right and left, respectively, were determined separately for the test periods following the contralateral (right-sided) and ipsilateral (left-sided) ablations for each animal. It was found that $61 \%$ of a total of 484 errors for all eight animals were made to the animals' right side following the right-sided removals; and $62 \%$ of a total of 73 errors were made to the animals' left side following the left-sided removals. There is therefore a tendency for the animals to prefer to respond to the cue situated on the side of the ablation. This trend fails to attain significance $\left(\chi^{2}=1 \cdot 33\right.$, 
1 degree of freedom) when the performance of all eight animals is analysed for the effect of both right- and left-sided removals; however, it attains significance $\left(\chi^{2}=33 \cdot 1, P=0.001\right)$ when only the performance subsequent to a right-sided ablation is considered for the six animals that were impaired after such a removal.

\section{DISCUSSION}

\section{EXTENT OF LESIONS}

It was our intention to spare the primary somatosensory projection area in animals which were to receive posterior parietal ablations. This intention, judged by macroscopic inspection of the surface lesions, was achieved in animals numbered 1 to 11 , except in the case of the right-sided lesion in animals Nos. 1 and 3 and the left-sided lesion in animals Nos. 5 and 7. Furthermore in none of six animals which were studied for thalamic changes was degeneration seen in the nucleus ventralis posterolateralis except in the case of animals Nos. 5 and 6 on the one side. Therefore the removals in the large majority of the animals in the present investigation are comparable to the posterior parietal ablations reported by other workers (e.g., Bates and Ettlinger, 1960).

\section{NEUROLOGICAL EXAMINATIONS}

On certain items of the neurological examination no animal gave evidence of a defect after surgery. To take only one example, there was no clear indication of a visual field defect in any animal.

There is little agreement concerning the typical response to plantar stimulation in the unoperated rhesus monkey (Denny-Brown and Chambers, 1958; Fulton and Keller, 1932; Rudolf, 1922). We were unable to establish that a single type of response occurs without exception or even predominantly before operation. The plantar reactions varied from animal to animal as greatly after posterior parietal removals as before surgery (Table II). Similarly, reactions to palmar touch varied as much after as before posterior removals, both from animal to animal and also within the individual animal. We are unable to relate the variability of the plantar and palmar reactions to any special test conditions.

Two neurological test items, visual and tactile placing, consistently gave evidence of changes in the contralateral limbs of animals with unilateral parietal removals. Both visual and tactile placing were absent or diminished on the contralateral side in the large majority of our animals. We were not able to find a clear relationship between loss of tactile placing and anterior removals or loss of visual placing and posterior removals as might have been expected (Denny-Brown and Chambers, 1958). Complete absence of the ipsilateral reactions was never observed, indicating a predominantly crossed defect as has been previously reported by Rademaker (1931) and Bard (1938).

Bates and Ettlinger (1960) suggested that the disorder of reaching for visual objects in monkeys with posterior parietal ablations may have its origin in a form of visual disorientation instead of a sensory ataxia. They gave various reasons for this view, emphasizing in particular the considerable accuracy with which the food that the animal had repeatedly missed was brought to the mouth once it was grasped. However, they were not in a position to observe reaching in animals with unilateral posterior parietal removals. In man a few authors (e.g., Brain, 1941) have described cases in which there was a disorder of reaching, with either hand, only to the side of the visual field contralateral to a parietal lesion. If visual disorientation in the monkey is similar to this condition in man then an animal with a unilateral posterior parietal removal would be expected to make errors with either hand in the contralateral but not in the ipsilateral visual field.

These expected results have not been obtained. For animals having unilateral removals reached inaccurately only with the contralateral hand, and then when the target was in either visual half field. Moreover, the animals with anterior parietal removals were more inaccurate than the animals having posterior ablations; and we were not able? to observe a reliable qualitative difference between the disability shown by animals respectively with anterior and posterior ablations. The degree of inaccuracy of visual reaching seemed to be associated in certain animals in which the posterior removals extended into the primary somatic area with the severity of certain other changes; for instance, animals Nos. 1 and 3 were slow and clumsy in their movements; they found it difficult to localize with the left (contralateral) hand a clip applied to the skin; and there was an abnormal posture and reduced movement in the limb which was inaccurate in reaching. On neurological examination of certain of the animals (Nos. 1, 2, 5, and 7) visual reaching was found to be more accurate than non-visual reaching; after one or both operations these animals were unable to find accurately with one hand a food object that had been placed in the other (restrained) hand and could not be seen, even though they reached accurately for food that could be seen.

These new findings do not lend support to the view that the disorder of reaching in parietal monkeys is a form of disorientation in the sense of Holmes 
(1918). Although we too have observed that both anterior and posterior parietal animals which repeatedly failed to grasp a small food object were eventually able to grasp it, and then immediately bring it accurately to the mouth in the affected hand, we suggest that the disorder of reaching is the result of a central disturbance of position sense. There are two reasons for this view: first the reaching for visual targets of animals with unilateral removals was inaccurate with the contralateral hand and not selectively in the contralateral visual field; secondly it has been shown by Ferraro and Barrera (1935) that anterior parietal ablations in the rhesus monkey give rise to a disorder of position sense in the contralateral limbs, and we can establish no difference, except in degree, between the defect of reaching following anterior and posterior ablations.

\section{QUANTITATIVE TESTS}

Contralateral posterior parietal ablations have been shown in the present investigation to cause significantly more disability than comparable ipsilateral removals on both of two tests performed with the left hand: on a test of tactile shape discrimination (performed in the dark) and on a test of the accuracy of reaching (performed in the light). Moreover when contralateral ablations were made in animals already having ipsilateral removals there was no significant additional impairment in excess of that resulting from single contralateral ablations; and similarly when ipsilateral ablations were added after inter-operative testing to contralateral removals, performance did not differ significantly from that following single ipsilateral ablations. Finally, the performance of three animals having one-stage bilaterally symmetrical posterior parietal ablations was somewhat less impaired than the performance of four animals having only one-sided contralateral ablations. These findings taken together suggest that there exists a predominantly crossed relationship between each posterior parietal region and the defect of tactile shape discrimination and of reaching with the contralateral hand. Such crossed effects for the placing reactions have already been reported by others (Denny-Brown and Chambers, 1958) and have been observed by us in animals having posterior parietal removals. However only in the work of Ruch and Fulton (1935) can we find earlier reference to the effects of unilateral posterior parietal ablations on somatosensory discriminations in the monkey. These authors describe one animal in which weight discrimination with the ipsilateral hand was not impaired following a unilateral posterior parietal ablation.

The crossed effects were not unexpected in our animals with anterior parietal ablations since the whole of the primary somatic projection area was removed. Our posterior parietal ablations, however, were placed in so-called 'association cortex'. For infero-temporal association cortex there is evidence (Ettlinger, 1959) of both crossed and uncrossed effects in the monkey, in this case upon visual discrimination: contralateral removals gave rise to significantly more impairment than ipsilateral removals but the greatest impairment resulted from bilateral (successive) removals. In the present study only crossed defects were found for tactile shape discrimination and for reaching, suggesting that there exists a large measure of independence between the hemispheres in the control of both of these activities. We have discussed elsewhere (Ettlinger and Kalsbeck, 1961) how to reconcile such a view (of the virtual independence of the left and right posterior parietal regions) with the contrary evidence of interaction, derived from transfer of tactile learning between the hands in normal monkeys.

The nature of the disorder of tactile shape discrimination in animals having posterior parietal ablations remains obscure. Bates and Ettlinger (1960) argued that it was secondary neither to loss of tactile sensibility nor to the disorder of reaching. Their view receives confirmation from our findings on the neurological examinations (that is of no somatosensory deficit with posterior lesions) and from our statistical correlations (that is, no significant correlation in animals Nos.1 to 11 between impairment of tactile shape discrimination and either the anterior extent of the ablations or the degree of inaccuracy of visual reaching). A new finding is the trend for animals with posterior removals to make more errors when the cue to which they should respond is placed on the side of the shelf contralateral to the most recent ablation. They seem to prefer to respond to the side of the shelf ipsilateral to the side of the most recent lesion.

Poor performance on the quantitative test for the accuracy of visual reaching may result from a genuine disorder of reaching, that is because the hand misses the pellet, and also from clumsiness of grasping in the presence of accurate reaching. Animals having posterior parietal ablations tended to miss the pellet altogether (if they made an error) and less frequently failed to take hold of it or knocked it off the belt through clumsiness. Animals having anterior parietal involvement (Nos. 12 and 14) also tended to miss the pellets (although animal No. 12 was initially accurate and became inaccurate only 10 days after surgery). However they were considerably more clumsy than the posterior animals in grasping and holding the pellet, if eventually the hand was placed on the pellet. There was only one 
animal (No. 12) in the present investigation that clearly showed a greater degree of inaccuracy of reaching for the tactile cues (the shapes $L$ and $T$ ) in the dark than in the light. This finding confirms the earlier observations of Bates and Ettlinger (1960).

Financial support of this work was provided by the research fund of the Institute of Neurology and by the Medical Research Council. To Dr. E. A. Carmichael we owe especial thanks for his interest and active encouragement of this investigation. We are also extremely grateful to Dr. M. C. Smith for help with the histological interpretations; to Dr. J. A. V. Bates who helped in the preparation of this paper; and to Miss $\mathrm{H}$. Shand for her accurate typing of the various versions.

\section{SUMMARY}

Fourteen rhesus monkeys were studied preoperatively in three ways: by neurological examination, by training with the left hand on a test of tactile shape discrimination (in the dark) and by testing for the accuracy of reaching with the left hand (in the light). Eight animals then received successive (left and then right, or conversely) posterior parietal ablations with inter-operative testing; three animals sustained bilateral removals of the same areas at a single operation; and three animals received anterior parietal ablations. The animals were again studied after surgery.

It was found that with unilateral removals both visual and tactile placing reactions were absent or diminished in the contralateral limbs; performance with the left hand on both the test of tactile shape discrimination and visual reaching was significantly more impaired following contralateral than ipsilateral posterior parietal removals; neither first nor second ipsilateral removals gave rise to unequivocal impairment on these tests; and the impairment of shape discrimination and reaching with the left hand following one-stage bilateral posterior parietal removals was no greater than that following unilateral right-sided removals.

These results indicate that defects of placing, tactile shape discrimination, and reaching associated with posterior parietal ablations in the monkey are predominantly crossed, and suggest that there exists a measure of independence between the hemispheres in the control of these activities. It is also argued that the disorder of reaching is the result of a central loss of position sense.

\section{REFERENCES}

Bard, P. (1938). Harvey Lect., 1937-8, Series 33, p. 143.

Bates, J. A. V., and Ettlinger, G. (1960). Arch. Neurol. (Chicago), 3, 177.

Brain, W. R. (1941). Brain, 64, 244.

Denny-Brown, D., and Chambers, R. A. (1958). Ass. Res. nerv. Dis. Proc., 36, 35.

Ettlinger, G. (1959). Brain, 82, 232.

(1962). Proc. Conf. Cerebral Dominance, Johns Hopkins Univ. Press, Baltimore.

$\longrightarrow$, and Kalsbeck, J. E. (1961). J. Physiol. (Lond.), 156, 34P.

Ferraro, A., and Barrera, S. E. (1935). Ass. Res. nerv. Dis. Proc., 15, 371 .

Fulton, J. F., and Keller, A. D. (1932). The Sign of Babinski. Baillière, Tindall \& Cox, London.

Holmes, G. (1918). Brit. J. Ophthal., 2, 449, 506.

Rademaker, G. G. J. (1931). Das Stehen. Springer, Berlin.

Ruch, T. C., and Fulton, J. F. (1935). Ass. Res. nerv. Dis. Proc., 15, 289.

Rudolf, G. de M. (1922). J. Neurol. Psychopath., 2, 337. 